\title{
Raltegravir in the management of HIV-infected patients
}

\author{
Hans-Jürgen Stellbrink \\ $\mathrm{ICH}$ and IPM Study Center Hamburg, \\ Hamburg, Germany
}

\begin{abstract}
Raltegravir has recently been licensed for the treatment of HIV-1 infection. Currently its use is limited to treatment-experienced patients and subjects with resistant virus. In addition to its activity in the setting of resistance and treatment failure, it appears to have great potential for first-line therapy and as a switch option for subjects with intolerance to other agents, as well. Overall tolerability in clinical trials was excellent, and the toxicity profile is non-overlapping with other agents, with no clear neuropsychiatric, gastrointestinal, or metabolic toxicity. Its metabolization occurs mainly via UGT1A1 rather than by the CYP450 system, resulting in a relatively unproblematic drug interaction profile. The independence of the compound from "boosting" of drug levels with ritonavir is an attractive feature for many patients suffering from ritonavir-associated side effects. However, it has to be dosed twice daily.

The unique effect of raltegravir on the establishment of viral latency makes it a logical component of treatment attempts aiming at reducing and controlling this viral sanctuary.

This review summarizes the clinical view on the role of this novel compound in HIV therapy.

Keywords: raltegravir, MK-0518, integrase inhibitor, HIV infection, HAART
\end{abstract}

Correpondence: Hans-Jürgen Stellbrink ICH and IPM Study Center Hamburg, Grindelallee 35, D-20I46 Hamburg, Germany

Tel +49404132420

Fax +49404I3242 22

Email stellbrink@ ich-hamburg.de
Until 2007, highly active antiretroviral therapy (HAART) was based on combinations of nucleoside (NRTI), nucleotide (NtRTI), non-nucleoside reverse transcriptase inhibitors (NNRTI), and protease inhibitors (PI), as well as the fusion inhibitor enfuvirtide. In late 2007 and early 2008, the CCR5 chemokine receptor blocker maraviroc and the first integrase inhibitor raltegravir were licensed in most countries, representing two new classes of drugs with novel modes of action.

Raltegravir as the first drug in its class targets HIV-1 integrase, an enzyme in the viral replication cycle that is essential for inserting HIV-1 proviral DNA into the host cell genome. In contrast to drugs from all other classes, this mode of action affects viral latency directly.

\section{Mechanism of action}

The complete viral life cycle requires integration of HIV-1 proviral DNA into the host cell genome. It occurs in three steps (Craigie 2001; Hazuda et al 2000; Lafemina et al 1992). The double-stranded DNA copy generated by reverse transcription of the HIV-1 viral RNA genome is associated with a "preintegration complex", containing both cellular and viral proteins, including integrase. In a first step, two nucleotides are removed from the 3 ' ends of the proviral DNA (see Figure 1). In a second step, the proviral DNA strands are inserted into the host DNA and joined with it. This process is termed "strand transfer". Subsequently, gaps in DNA are repaired by cellular enzymes by removing the two unpaired nucleotides at the 5' end of the proviral DNA. HIV integrase catalyses the first two steps.

With the discovery of selective inhibitors of strand transfer, which share a ß-diketo acid moiety, orally bioavailable agents came within the reach (Hazuda et al 1999, 


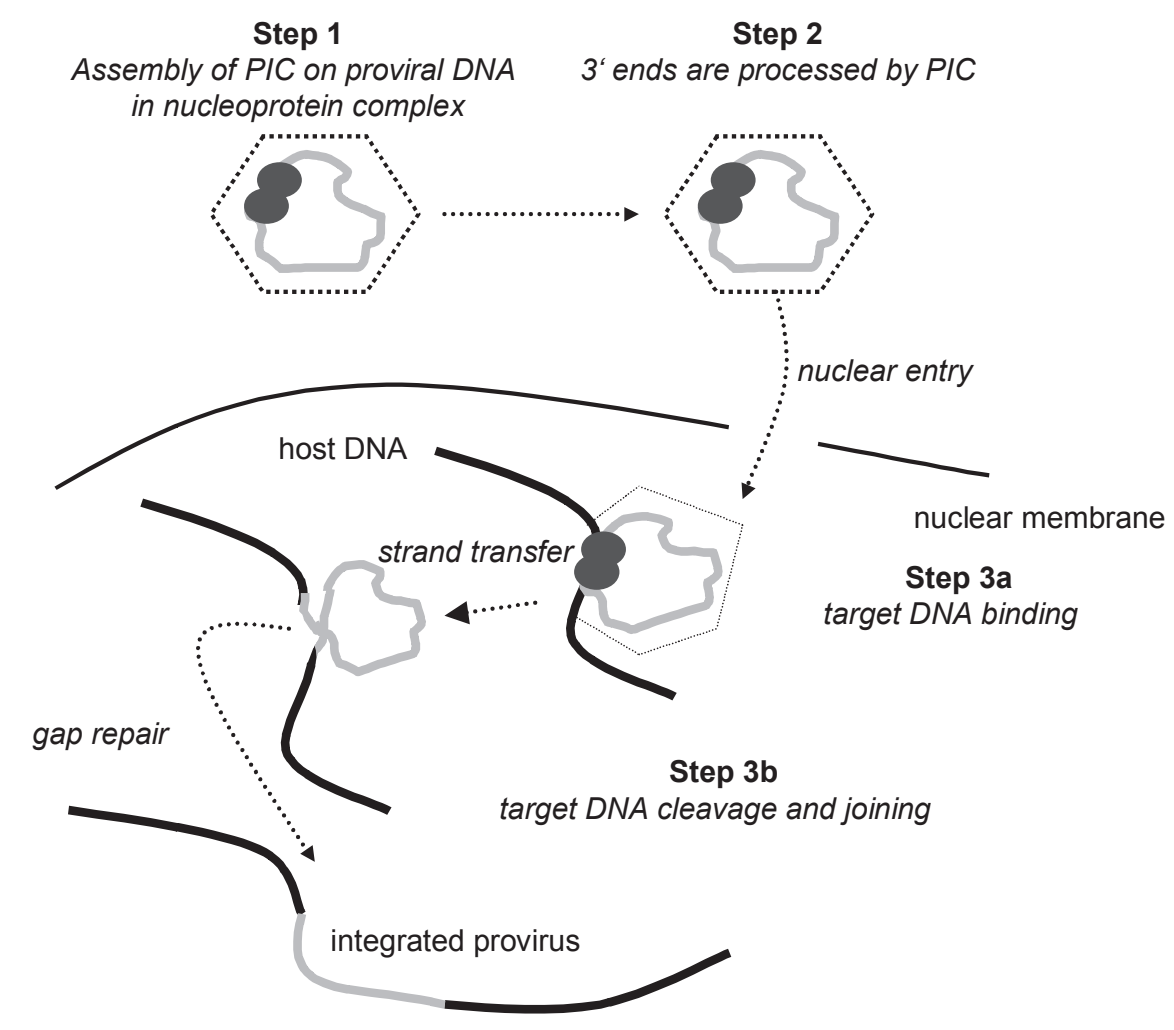

Figure I Steps of viral integration. Adapted with permission from: New Classes of Antiretrovirals: The Potential Clinical Role of Integrase Inhibitors and Entry Inhibitors. Clinical Care Options; Reston,Virginia; Slideset accessed at clinicaloptions.com/hiv. Abbreviation: PIC, prointegration complex.

2004; Espeseth et al 2000; Pais et al 2002; Embrey et al 2005). Chemical optimisation of these compounds led to naphthyridine derivatives and L-900612 or MK-0518 (raltegravir) as a promising candidate compound.

\section{Pharmacological properties, metabolism}

Oral absorption of raltegravir is rapid, and oral bioavailability approaches $32 \%$. Plasma protein binding reaches $83 \%$. Raltegravir has a rapid initial half-life of $\sim 1$ hour and a terminal half-life of $\sim 7$ hours (Merck and Co. 2007), not supporting once daily administration. Steady state pharmacokinetics are reached within 2 days after the first dose (Iwamoto et al 2008b).

Although a high fat meal postpones the maximum plasma concentration by approximately 7.5 hours and decreases it by $34 \%$, this effect is outweighed by an augmentation of exposure over time, as assessed by an increase of the area under the curve (AUC) by 19\%. As a consequence, no specific restrictions are recommended with regard to food.

In contrast to most other antiretroviral drugs, raltegravir is metabolized by glucuronidation via UGT1A1 (Kassahun et al 2006, 2007; Merck and Co. 2007, 2008). Excretion in feces $(51 \%)$ and in urine $(31 \%)$ accounts for most of the elimination. No dose adjustment is required for gender, age, hepatic or renal function, or body mass index.

\section{Drug interactions}

Raltegravir neither induces nor inhibits cytochrome P450 enzymes, nor is it a substrate (Iwamoto et al 2008a). Therefore, few interactions have to be expected with other drugs metabolized via the P450 enzyme system, such as protease inhibitors (PI), non-nucleoside reverse transcriptase inhibitors (NNRTI), maraviroc or frequent concomitant medication (eg, anticonvulsants, lipid-lowering drugs).

Of note, coadministration of raltegravir with tipranavir/ ritonavir led to a $55 \%$ decrease in raltegravir $\mathrm{C}_{12 \mathrm{~h}}$ trough levels (Wenning et al 2006b), while reducing maximum concentrations $\left(\mathrm{C}_{\max }\right)$ and the $\mathrm{AUC}_{0-12 \mathrm{~h}}$ less markedly (24 and $18 \%)$.

Indinavir and Atazanavir inhibit UGT1A1. Because Indinavir is used rarely due to its specific toxicity, atazanavir is a likely combination partner for raltegravir. Pharmacokinetic studies with atazanavir, with or without ritonavir-"boosting", 
showed a 30\%-70\% increase in raltegravir AUC (Mistry et al 2007). In view of its good tolerability (see below), this is probably not clinically relevant, and no dose adjustment is required for either drug. No relevant interaction was observed with efavirenz, etravirine, or tenofovir, either (Iwamoto et al 2006b; Wenning et al 2006a). Rifampicin, however, reduces raltegravir concentrations at 12 hours by $61 \%$ (Iwamoto et al 2006a), which appears clinically relevant. Combinations of raltegravir with rifampicin or similarly potent inducers should therefore be avoided where possible (Kassahun et al 2006, 2007). Importantly, there appears to be no relevant interaction with contraceptives (Anderson et al 2007).

\section{Antiviral activity in vitro}

Marked and broad in vitro antiviral activity was observed against HIV-1 variants sensitive or resistant to NRTIs, NNRTIs, and PIs, as well as against SIV (Miller et al 2006). Despite considerable (40\%) heterogeneity between HIV-1 and HIV-2 integrase genes, clinical HIV-2 isolates also show a high sensitivity to raltegravir (Roquebert et al 2008).

\section{Clinical trials in HIV-infected patients}

For a list of relevant trials and a brief summary of their results, see Table 1.

\section{Phase II}

In protocol 004 (Markowitz et al 2007), doses of 100, 200, 400 , and $600 \mathrm{mg}$ of raltegravir twice daily were compared to placebo (part 1) or efavirenz $600 \mathrm{mg}$ (part 2) in antiretroviral naïve subjects in a randomized, double-blind manner, stratified for initial HIV plasma viremia $(<$ or $>50000$ copies/mL). Minimum CD4 cell count at screening was $100 / \mu \mathrm{L}$, minimum viral load 5000 copies/mL. Thirty-five subjects entered part 1 and received a 10-day course of raltegravir monotherapy at the doses described above or placebo. Baseline characteristics were not significantly different between the study arms. The mean decrease at the different raltegravir doses after 10 days of monotherapy was 1.66 to $2.16 \log _{10}$ copies/mL, as compared with placebo $(-0.17$ $\log _{10}$ copies/mL), with no significant difference between the raltegravir dosage arms.

Of these trial participants, 30 entered the second part, together with 198 additional subjects. They were randomized to receive either raltegravir at the doses mentioned above, or efavirenz (EFV) $600 \mathrm{mg}$, all in conjunction with tenofovir (TDF) and lamivudine (3TC). Baseline characteristics were comparable between the groups, with plasma viremia ranging between 4.6 and $4.8 \log _{10}$ copies/mL and CD4+ T-cells from 271 to 314 cells $/ \mathrm{mm}^{3}$. None of the study subjects had primary resistance to TDF, 3TC, or EFV. Viral load

Table I Clinical trial results with Raltegravir (MK-05 I8)

\begin{tabular}{|c|c|c|c|c|}
\hline Protocol & Study Design & Patient population & Treatment & Results \\
\hline 004 & $\begin{array}{l}\text { Multicenter, } \\
\text { double-blind, } \\
\text { randomized, } \\
\text { controlled, } \\
\text { dose-escalating }\end{array}$ & $\begin{array}{l}\text { antiretroviral naive } \\
(\mathrm{n}=198)\end{array}$ & $\begin{array}{l}\text { RGV I00, 200, } 400 \text {, or } 600 \mathrm{mg} \\
\text { bid vs efavirenz } 600 \mathrm{mg} \text { qd } \\
\text { (all + lamivudine and } \\
\text { tenofovir) }\end{array}$ & $\begin{array}{l}\text { week } \mathbf{2 4} \\
\text { pVL }<\mathbf{5 0} \text { copies/mL: } 85 \%-95 \% \\
\text { (RGV arms) vs } 92 \% \text { (efavirenz arm), } \mathrm{p}=\text { n.s. } \\
\text { CD4+ T-cells }(/ \mu \mathrm{L}):+139-175 \\
\text { (RGV arm) vs }+\mathrm{II} 2 \text { (efavirenz) } \mathrm{p}=\text { n.s. } \\
\text { week } \mathbf{4 8} \\
\text { pVL }<\mathbf{5 0} \text { copies/mL: response } \\
\text { sustained at } 83 \%-88 \% \text { across all arms }\end{array}$ \\
\hline 005 & $\begin{array}{l}\text { Multicenter, } \\
\text { double-blind, } \\
\text { randomized, } \\
\text { controlled, } \\
\text { dose-escalating }\end{array}$ & $\begin{array}{l}\text { treatment-experienced, } \\
\text { multidrug-resistant } \\
(\mathrm{n}=\mathrm{I78})\end{array}$ & $\begin{array}{l}\text { RGV } 200,400 \text {, and } 600 \mathrm{mg} \\
\text { bid vs placebo (+ optimized } \\
\text { background regimen in all) }\end{array}$ & 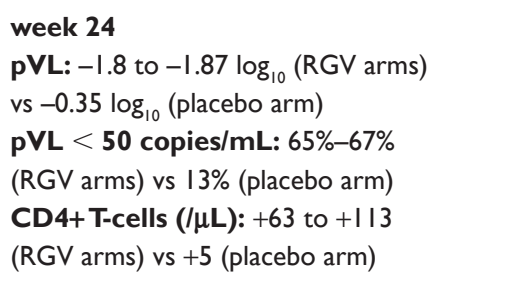 \\
\hline $\begin{array}{l}\text { BENCHMRK-I } \\
+ \\
\text { BENCHMRK-2 }\end{array}$ & $\begin{array}{l}\text { Phase III, parallel, } \\
\text { multinational, } \\
\text { randomized, } \\
\text { double-blind, } \\
\text { placebo-controlled } \\
\text { studies }\end{array}$ & $\begin{array}{l}\text { treatment-experienced, } \\
\text { multidrug-resistant } \\
(\mathrm{n}=350 ; \mathrm{n}=349)\end{array}$ & $\begin{array}{l}\text { RGV } 400 \text { mg bid vs placebo } \\
\text { (+ optimized background } \\
\text { regimen in all) }\end{array}$ & $\begin{array}{l}\text { week } 16 \text { (primary end-point) } \\
\text { pVL }<\mathbf{4 0 0} \text { copies/mL: } 77 \% \text { and } 77 \% \\
\text { (RGV arm) vs } 41 \% \text { and } 43 \% \text { in placebo arm } \\
\text { pVL }<\mathbf{5 0} \text { copies per } \mathbf{m L :} 61 \% \text { and } 62 \% \\
\text { (RGV arm) vs } 33 \text { and } 36 \% \text { in placebo arm } \\
\text { CD4+ T-cells }(/ \mu L):+83 \text { and }+86 \\
\text { (RGV arm) vs }+3 I \text { and }+40 \text { in placebo arm }\end{array}$ \\
\hline
\end{tabular}

Abbreviations: RGV, raltegravir; $\mathrm{PVL}$, plasma viral load. 
reduction to less than 50 copies of HIV RNA per mL was more rapid and significantly more frequent at weeks 4 and 8 in all raltegravir arms, but response was not different at any time-point thereafter up to and including week 48. CD4+ $\mathrm{T}$-cells increased to a comparable extent in all arms. Adverse events were infrequent, of mild to moderate intensity, with no obvious difference between the treatment arms. Continued follow-up until week 96 confirmed the sustained efficacy of raltegravir in this study (Markowitz et al 2008).

Protocol 005 (Grinsztejn et al 2007) was a multicenter, randomized, double-blind, placebo-controlled dose-ranging trial in treatment-experienced patients. 178 patients with greater than 5000 HIV RNA copies per mL, a minimum of 50 CD4+ T-cells on 3 months of stable antiretroviral therapy, and resistance to at least 3 drug classes were included and analyzed. Investigators selected an optimal background regimen according to all available resistance test results. Randomization was performed in a 1:1:1:1 fashion to raltegravir doses of 200, 400, and $600 \mathrm{mg}$ twice daily, or placebo, stratified by PI resistance at baseline and prior enfuvirtide use. Primary end-points were week 24 response and toxicity.

Virological response was significantly better for all raltegravir arms as compared to placebo, with no significant difference between the raltegravir arms. This difference in favour of raltegravir was observed with any extent of resistance to the compounds of the background regimen, as assessed by genotypic or phenotypic sensitivity scores (GSS or PSS), ie, the number of drugs a patient received, which were predicted to be active at baseline. Use of additional enfuvirtide was also associated with a better virological outcome. There was no obvious difference of toxicity profile for any raltegravir dose in comparison to placebo.

\section{Phase III}

Based on the results of the aforementioned trials, $400 \mathrm{mg}$ twice daily was selected as a dose for the phase III studies.

MK-0518-018 and MK-0518-019 (BENCHMRK-1 and BENCHMRK-2 (Cooper et al 2007; Steigbigel et al 2007) were two parallel, double-blind, placebo-controlled studies. In both trials, an investigator-selected, resistance analysisbased optimal regimen was combined with either raltegravir or placebo. Randomization was performed in a 2:1 manner. Patients were required to have triple-class resistant virus at baseline and HIV plasma viremia greater than 1000 copies per mL. BENCHMRK-1 was conducted in Europe, Asia, the Pacific, and Peru, BENCHMRK-2 in the Americas. The primary end-point was viral suppression to $<400$ copies per $\mathrm{mL}$ at week 16 , with virus suppression to $<50$ copies per $\mathrm{mL}$ and change from baseline viral load and CD4+ T-cell count evaluated as secondary end-points.

Baseline demographic variables and patient characteristics were comparable between the study arms and the studies.

The raltegravir arms in both trials were superior over placebo with regard to all end-points. The difference was maintained irrespective of the extent of baseline resistance (as assessed by GSS and PSS), baseline HIV RNA levels, and baseline CD4+ T-cell count. There was no significant difference with respect to toxicity between the study arms.

A superior response was sustained until week 24 in a combined analysis of both trials. The difference in favour of raltegravir persisted regardless of the background regimen. A repeat analysis after 48 weeks of follow-up confirmed the week 24 results: The response rate (HIV RNA $<50$ copies per $\mathrm{mL}$ ) in the raltegravir group at week 48 was $89 \%$ in combination with enfuvirtide and darunavir, when both were used for the first time (Cooper et al 2008). This approaches response rates in previously untreated patients and therefore represents a marked improvement for subjects with resistant virus.

\section{Toxicity}

Study 005 and the BENCHMRK studies are more difficult to interpret with respect to toxicity, since all participants received complex regimens with a high potential of untoward reactions, eg, gastrointestinal side effects associated with ritonavir-boosted protease inhibitors. However, side effects and laboratory abnormalities were balanced between the groups in both trials, with no significant difference between the study arms. In this regard, MK-0518-004 provides a clearer picture, because there was a 10-day run-in monotherapy phase. Moreover, subsequently all subjects received a background regimen comprising of only tenofovir and lamivudine. From the 004 and 005 studies (Grinsztejn et al 2007; Markowitz et al 2007), diarrhea, nausea, fatigue, and muscle aches were reported. Laboratory abnormalities include increases in pancreatic amylase and hepatic transaminase levels. Of note, no lipid abnormalities were reported from the 004 study (Markowitz et al 2007).

When analyzing the phase II and III trials together, only a few patients on raltegravir or placebo discontinued for adverse events. Adverse events were mostly mild to moderate. Headache, nausea, and diarrhea were the most common treatment related adverse events occurring in $>10 \%$ of subjects. They were evenly distributed between the arms.

Fatigue (7.9\% vs $4.6 \%$ ), herpes zoster ( $4.1 \%$ vs $0.7 \%$ ), nasopharyngitis (6.1\% vs $3.9 \%)$, and mild to moderate rash 
(5.3\% vs $2.5 \%)$ not requiring treatment discontinuation, as well as elevated creatine phosphokinase (CPK, 3.7\% vs $1.1 \%$ ) were observed more frequently on raltegravir ( $>2 \%$ difference). Myopathy and rhabdomyolysis were also reported, but the causal role of raltegravir in these instances is unclear. It is therefore recommended that raltegravir be used with caution in combination with other drugs associated with muscle toxicity.

Overall, more hepatic laboratory events were observed on raltegravir. However, no clear difference was noted with respect to other hepatic adverse events in the total study population or within the subgroup of hepatitis B or C coinfected subjects.

In the pooled analysis, a higher rate of malignancies was observed in patients on raltegravir $(2.5 \%$ vs $1.5 \%$ for placebo). After adjustment for duration of exposure, however, the adjusted risk of malignancy per 100 patientyears was not significantly different with a rate of 2.32 for raltegravir and 1.92 for the comparator groups (relative risk 1.209, 95\% confidence interval 0.44-4.14). Moreover, the malignancies occurred in subjects with prior AIDS and were very heterogeneous, with 8/19 in the raltegravir groups representing recurrent disease and the others occurring within three months of enrolment. This indicates no causal relationship to raltegravir (Merck and Co 2007).

A recent report of four cases suggests that raltegravir might lead to exacerbation of depression in subjects on psychotropic medication for mental disorders (Harris et al 2008a), possibly due to drug-drug interactions. This observation, however, remains to be substantiated.

Raltegravir is currently classified as FDA pregnancy category $\mathrm{C}$, mainly due to lack of data on reproductive toxicity.

\section{Resistance}

Knowledge about resistance selection patterns for raltegravir is rapidly expanding, and they are yet understood incompletely. Several characteristic mutations leading to typical amino acid exchanges were characterized in cell culture studies and confirmed in clinical samples from trial participants with virological failure on raltegravir (Merck and Co 2007). The Q148K mutation was typically followed by an amino acid substitution at other positions (E138A, G140A, and V54I), with additional mutations developing at higher drug concentrations. Insertional mutagenesis confirmed the role of these amino acid changes. The Q148K, E138A/Q148K, and E138A/G140A/Q148K resulted in a substantial foldshift (46- to 508-fold) in raltegravir IC50. Another study identified the E92Q, G140S Q148H, N155H, and E157Q as key mutations (Malet et al 2008). Furthermore, virus carrying the Q148K as well as the E138A, G140A, and V54I changes was highly cross-resistant to elvitegravir and had a significantly reduced sensitivity against other integrase inhibitors in development (Goethals et al 2008). In another study, mutated integrase enzymes displayed an impairment of enzyme function (Malet et al 2008), indicating reduced replicative fitness. The clinical relevance of this observation, however, remains to be demonstrated. In vitro and in vivo data correspond very well, as the majority of patients in the BENCHMRK trials with virological failure on raltegravir, in whom genotyping was performed, exhibited mutations in integrase known to be associated with raltegravir resistance (Cooper et al 2008). This reflects the strong selective pressure of the drug and its barrier to resistance, which appears lower than for ritonavir-boosted protease inhibitors, but somewhat higher than for first-generation NNRTIs.

Subjects failing on raltegravir in clinical trials exhibited two pathways to resistance. The virus appears to reach a moderate level of resistance (10- to 25-fold) by acquiring a change at position Q148 (H,K, or R) or the N155H. Other mutations, which by themselves have only limited impact on resistance (L74M, E92Q,T97A, E138K, G140S, V151I, G163G/R, and $\mathrm{D} 232 \mathrm{D} / \mathrm{N})$, appear to decrease susceptibility further in conjunction with either of the two (Hazuda et al 2007).

The observation of at least partial in vitro cross-resistance between raltegravir and elvitegravir, another integrase inhibitor in phase III studies, was substantiated by clinical case studies. Two participants of an elvitegravir phase II trial switched to raltegravir upon failure to the study drug. No virological response was noted within one week. One subject harboured virus carrying the Q148R key raltegravir mutation in conjunction with others; the integrase sequence of the other one was not amplifiable (DeJesus et al 2007).

With respect to activity against different HIV clades, a recent study found no impact of clade-specific integrase polymorphisms on raltegravir sensitivity (van Baelen et al 2008).

\section{Therapeutic potential}

Raltegravir as the first of its class has demonstrated high antiviral activity in a series of clinical trials, which have led to its approval for treatment-experienced subjects. Its optimal use in this setting is becoming increasingly clear: a subgroup analysis of the pooled BENCHMRK study data shows that response rates at week 48 were highest when the regimen comprised two other components for which sensitivity was predicted (Cooper et al 2008). Of note, 
response rates were not higher when more than two active components were combined with raltegravir. Therefore, three active components (which include raltegravir) achieve high response rates in treatment-experienced patients, which in fact are similar to those in previously untreated subjects.

Raltegravir also represents an important option for patients who do not tolerate an active agent in their combination, while exhibiting optimal response. In a series of 35 subjects responding to a regimen comprising enfuvirtide, enfuvirtide was replaced by raltegravir, while the rest of the regimen was left unchanged. Virological suppression was sustained until a median of 7 months of follow-up (Harris et al 2008b). The strategy of replacing active components by raltegravir is currently being investigated in several trials.

A large phase III trial currently addresses the activity of raltegravir in first-line therapy. It is compared with the current standard-of-care efavirenz, both in combination with tenofovir plus emtricitabine (trial 021). Results are expected for the end of 2008.

It is unclear if the more rapid increase in response rates in the raltegravir arms in study 004 (Murray et al 2007) in comparison with the efavirenz arm indicates higher antiviral potency. Mathematical modelling actually suggests that calculations of viral clearance rates during early therapy depend on the mode of action of the drugs employed, ie on the stage of the viral life-cycle at which they interfere, and that this appears to make a difference only early in treatment (Sedaghat et al 2008). This actually challenges previous pathophysiological interpretations of studies on viral clearance rates. Should recently infected cells with unintegrated proviral DNA, which proceed to integration and virus production in the early phase of HAART, contribute a lot to plasma viral RNA in this phase, raltegravir might help a lower number of infected cells to be reached before later phases of viral decay are entered. That might not make a difference for viral RNA suppression in these later phases, but may reduce the viral depository of quasi-species and the basis for re-activation of virus from the latent pool. This view, however, is speculative and remains hypothetical at present.

Another very positive feature of the drug is its independence from pharmacological "boosting" with ritonavir. This makes it a very attractive option for patients with gastrointestinal side effects on ritonavir-boosted PIs and/or intolerance to efavirenz or nevirapine, as well as for patients in whom lipid abnormalities cannot be treated effectively during PI therapy. Several switch studies investigating improvement of neuropsychiatric toxicity of efavirenz or lipid and gastrointestinal toxicity on a ritonavir-boosted PI are ongoing.

Of the few relevant pharmacokinetic interactions, the inhibition of the metabolising enzyme UGT1A1 by atazanavir might even be exploited therapeutically. The concept of "boosting" raltegravir levels in order to circumvent twice daily dosing as one of the few disadvantageous features of the drug will be investigated in a clinical trial.

The recent approval of raltegravir and other compounds active against triple-class resistant virus has been a breakthrough for patients with uncontrolled replication due to resistance. The term "paradigm shift" is frequently employed for this change in therapeutic perspectives. The rapid appearance of resistance with the associated mutational changes in case of failure on raltegravir, however, demonstrates that suboptimal use of this novel compound (ie, in the context of too few active drugs) could ultimately lead to a similar rescue therapy situation. The BENCHMRK studies suggest that two other active drugs provide the necessary background for achieving the optimal effect of raltegravir.

For HIV-2, the number of treatment options unfortunately is far more limited than for HIV-1. In contrast to NNRTIs and some PIs, raltegravir appears to have comparable activity against HIV-2. Controlled clinical data, however, are still lacking. The same applies to pediatric application.

\section{Ongoing or planned clinical trials}

Clinical trials are underway to investigate a switch from enfuvirtide or a ritonavir-boosted protease inhibitor, a replacement of nucleoside analogues in a PI-based combination, alternative first-line combinations of lopinavir/ritonavir and raltegravir or raltegravir and tenofovir/emtricitabine, as well as abacavir/lamivudine and raltegravir.

The unique effect of raltegravir on viral integration prompted clinical studies of its impact on viral latency by adding raltegravir to a fully suppressive combination or combining it with the histone deacetylase inhibitor valproic acid. Furthermore, it is being investigated in combination with tenofovir/emtricitabine for non-occupational postexposure prophylaxis. Other studies address children, African patients and acutely infected subjects, as well as a multitude of pharmacokinetic questions.

Raltegravir provides an important improvement of the therapeutic armamentarium against HIV-1 and probably HIV-2. Future studies will show if its high antiviral activity and its effect on the establishment of viral latency 
represent the desired step forward towards life-long control of HIV.

\section{Disclosures}

The author has served as a scientific advisor to Merck.

\section{References}

Anderson MS, Wenning LA, Moreau A, et al. 2007, Effect of raltegravir (RAL) on the pharmacokinetics (PK) of oral contraceptives [abstract]. 47th Annual Interscience Conference on Antimicrobial Agents and Chemotherapy (ICAAC), 17-20 September 2007, Chicago, IL, USA abstract A-1425. 2007.

Cooper DA, Gatell J, Rockstroh J, et al. 2007. Results of BENCHMRK-1, a Phase III study evaluating the efficacy and safety of MK-0518, a Novel HIV-1 integrase inhibitor, in patients with triple-class resistant virus [abstract]. 14th Conf Retro Opportun Infect Los Angeles abstract 105aLB.

Cooper DA, Steigbigel RT, Gatell JM, et al. 2008. Subgroup and resistance analyses of raltegravir for resistant HIV-1 infection. $N$ Engl J Med, 359:355-65.

Craigie R. 2001. HIV integrase, a brief overview from chemistry to therapeutics. $J$ Biol Chem, 276:23213-16.

DeJesus E, Cohen C, Elion R, et al. 2007. First report of raltegravir (RAL, MK-0518) use after virologic rebound on elvitegravir (EVT, GS 9137) [abstract]. 4th IAS Conference on HIV Pathogenesis, Treatment and Prevention, Sydney, Australia, 22-25, July 2007; Abstract TUPEB032. 2007.

Embrey MW, Wai JS, Funk TW, et al. 2005. A series of 5-(5,6)-dihydrouracil substituted 8-hydroxy-[1,6]naphthyridine-7-carboxylic acid 4-fluorobenzylamide inhibitors of HIV-1 integrase and viral replication in cells Bioorg Med Chem Lett, 15:4550-4.

Espeseth AS, Felock P, Wolfe A, et al. 2000. HIV-1 integrase inhibitors that compete with the target DNA substrate define a unique strand transfer conformation for integrase. Proc Natl Acad Sci U S A, 97:11244-9.

Goethals O, Clayton R, Wagemans E, et al. 2008. Resistance mutations in HIV-1 integrase selected with raltegravir or elvitegravir confer reduced susceptibility to a diverse panel of integrase inhibitors [abstract]. XVII International HIV Drug Resistance Workshop June 10-14, 2008 Sitges, Spain abstract no. 2008.

Grinsztejn B, Nguyen BY, Katlama C, et al. 2007. Safety and efficacy of the HIV-1 integrase inhibitor raltegravir (MK-0518) in treatmentexperienced patients with multidrug-resistant virus: a phase II randomised controlled trial. Lancet, 369:1261-9.

Harris M, Larsen G, Montaner JS. 2008a. Exacerbation of depression associated with starting raltegravir: a report of four cases. AIDS, 22:1890-2.

Harris M, Larsen G, Montaner JS. 2008b. Outcomes of multidrug-resistant patients switched from enfuvirtide to raltegravir within a virologically suppressive regimen. Aids, 22:1224-6.

Hazuda D, Blau CU, Felock P, et al. 1999. Isolation and characterization of novel human immunodeficiency virus integrase inhibitors from fungal metabolites. Antivir Chem Chemother, 10:63-70.

Hazuda D, Miller MD, Nguyen BY, et al. 2007. Resistance to the HIVintegrase inhibitor raltegravir: analysis of Protocol 005, a Phase 2 study in patients with triple-class resistant HIV-1 infection [abstract]. Antivir Ther, abstract no.8[12], S10.

Hazuda DJ, Anthony NJ, Gomez RP, et al. 2004. A naphthyridine carboxamide provides evidence for discordant resistance between mechanistically identical inhibitors of HIV-1 integrase. Proc Natl Acad Sci U S A, 101:11233-8.

Hazuda DJ, Felock P, Witmer M, et al. 2000. Inhibitors of strand transfer that prevent integration and inhibit HIV-1 replication in cells. Science, 287:646-50.
Iwamoto M, Kassahun K, Troyer MD, et al. 2008a. Lack of a pharmacokinetic effect of raltegravir on midazolam: in vitro/in vivo correlation. J Clin Pharmacol, 48:209-14.

Iwamoto M, Wenning LA, Liou SY, et al. 2006a. Rifampin (RIF) modestly reduces plasma levels of MK-0518 [abstract]. 8th international congress on drug therapy in HIV infection, Glasgow, United Kingdom, November 12-16, 2006; abstract P299.

Iwamoto M, Wenning LA, Petry AS, et al. 2006b. Minimal effect of ritonavir (RTV) and efavirenz (EFV) on the pharmacokinetics (PK) of MK-0518 [abstract]. 46th Interscience Conference on Antimicrobial Agents and Chemotherapy; September 27-30, 2006; San Francisco, USA abstract A-373.

Iwamoto M, Wenning LA, Petry AS, et al. 2008b. Safety, tolerability, and pharmacokinetics of raltegravir after single and multiple doses in healthy subjects. Clin Pharmacol Ther, 83:293-99.

Kassahun K, McIntosh I, Cui D, et al. 2007. Metabolism and disposition in humans of raltegravir (MK-0518), an anti-AIDS drug targeting the human immunodeficiency virus 1 integrase enzyme. Drug Metab Dispos, 35:1657-63.

Kassahun K, McIntosh I, Hreniuk D, et al. Absorption, metabolism and excretion of MK-0518, a potent HIV-1 integrase inhibitor, in healthy male volunteers [abstract]. 46th Interscience Conference on 1 Agents and Chemotherapy; September 27-30, 2006; San Francisco, USA abstract A-372.

Lafemina RL, Schneider CL, Robbins HL, et al. 1992. Requirement of active human immunodeficiency virus type 1 integrase enzyme for productive infection of human T-lymphoid cells. J Virol, 66:7414-19.

Malet I, Delelis O, Valantin MA, et al. 2008. Mutations associated with failure of raltegravir treatment affect integrase sensitivity to the inhibitor in vitro. Antimicrob Agents Chemother, 52:1351-8.

Markowitz M, Nguyen BY, Gotuzzo E, et al. 2008. Sustained antiretroviral efficacy of raltegravir as part of combination ART in treatment-naive HIV-1 infected patients: 96-week data [abstract]. 17th International AIDS Conference; August 3-8, 2008; Mexico City, Mexico Abstract TUAB0102.

Markowitz M, Nguyen BY, Gotuzzo E, et al. 2007. Rapid and durable antiretroviral effect of the HIV-1 Integrase inhibitor raltegravir as part of combination therapy in treatment-naive patients with HIV-1 infection: results of a 48-week controlled study. J Acquir Immune Defic Syndr, 46:125-33.

Merck and Co., W. S. N. 0. U. Isentress ${ }^{\mathrm{TM}}$ FDA Briefing Document. Food And Drug Administration Homepage. 2007. www.fda.gov/ohmrs/ dockets/ac/07/briefing/2007-4314b1-01-Merck.pdf.

Merck and Co., W. S. N. 0. U. Isentress ${ }^{\mathrm{TM}}$ Prescribing Information. US Food and Drug Administration Homepage. 2008. www.fda.gov/cder/ foi/label/2007/0221451b1.pdf.

Mistry G, Wenning L, Petry Aet al. 2007. Atazanavir modestly increases plasma leves of MK-0518 [abstract]. 4th IAS Conference on HIV Pathogenesis, Treatment and Prevention, Sydney, Australia, 22-25, July 2007; abstract 1985

Murray JM, Emery S, Kelleher AD, et al. 2007.Antiretroviral therapy with the integrase inhibitor raltegravir alters decay kinetics of HIV, significantly reducing the second phase/Aids, 21:2315-21.

Pais GC, Zhang X, Marchand C, et al. 2002. Structure activity of 3-aryl-1,3-diketo-containing compounds as HIV-1 integrase inhibitors. J Med Chem, 45:3184-94.

Roquebert B, Damond F, Collin G, et al. on behalf of the French ANRS HIV2008. HIV-2 integrase gene polymorphism and phenotypic susceptibility of HIV-2 clinical isolates to the integrase inhibitors raltegravir and elvitegravir in vitro. $J$ Antimicrob Chemother, DOI 10.1093/jac/dkn335.

Sedaghat AR, Dinoso JB, Shen L, et al. 2008. Decay dynamics of HIV-1 depend on the inhibited stages of the viral life cycle. Proc Natl Acad Sci U S A, 105:4832-7.

Steigbigel R, Kumar P, Eron J, et al. 2007. Results of BENCHMRK-2, a Phase III study evaluating the efficacy and safety of MK-0518, a novel HIV-1 integrase inhibitor, in patients with triple-class resistant virus [abstract]. 14th Conf Retro Opportun Infect Los Angeles abstract 105bLB. 
van Baelen K, van Eygen V, Rondelez E, et al. 2008. Clade-specific HIV-1 integrase polymorphisms do not reduce raltegravir and elvitegravir phenotypic susceptibility Aids, Epub ahead of print.

Wenning LA, Friedman E, Kost JT, et al. 2006a. Lack of a significant drug interaction between MK-0518 and tenofovir disoproxil fumarate (TDF) [abstract]. 46th Interscience Conference on Antimicrobial Agents and Chemotherapy; September 27-30, 2006; San Francisco, USA abstract A-375.
Wenning LA, Hanley H, Stone J, et al. 2006b. Effect of tipranavir + ritonavir (TPV + RTV) on pharmacokinetics of MK-0518 [abstract]. 46th Interscience Conference on Antimicrobial Agents and Chemotherapy; September 27-30, 2006; San Francisco, USA abstract A-374. 2006 b. 